\title{
Transcriptome analyses of Anguillicola crassus from native and novel hosts
}

Anguillicola crassus is a swim bladder nematode of eels. The parasite is native to the Asian eel Anguilla japonica, but was introduced to Europe and the European eel Anguilla anguilla in the early 1980s. A Taiwanese source has been proposed for this introduction. In the new host in the recipient area, the parasite appears to be more pathogenic. As a reason for these differences, genetically fixed differences in infectivity and development between Taiwanese and European A.crassus have been described and disentangled from plasticity induced by different host environments. To explore whether transcriptional regulation is involved in these lifecycle differences, we have analysed a "common garden", cross infection experiment, using deep-sequencing transcriptomics. Surprisingly, in the face of clear phenotypic differences in life history traits, we identified no significant differences in gene expression between parasite populations or between experimental host species. From 120,000 SNPs identified in the transcriptome data we found that European A. crassus were not a genetic subset of the Taiwanese nematodes sampled. The loci that have the major contribution to the European-Taiwanese population differentiation show an enrichment of synonymous and non-coding polymorphism. This argues against positive selection in population differentiation. However, genes involved in protein processing in the endoplasmatic reticulum membrane and genes bearing secretion signal sequences were enriched in the set of genes most differentiated between European and Taiwanese $A$. crassus. These genes could be a source for the phenotypically visible genetically fixed differences between European and Taiwanese A. crassus. 
2 Emanuel Heitlinger ${ }^{1}$, Horst Taraschewski ${ }^{2}$, Urszula Weclawski ${ }^{2}$, Karim Gharbi ${ }^{3,4}$ 3 and Mark Blaxter ${ }^{3,4}$

41 Department for Molecular Parasitology, Institute for Biology, Humboldt 5 University Berlin, Philippstrasse 13, Haus 14, Berlin, Germany

62 Department of Ecology and Parasitology, Zoological Institute, Karlsruhe 7 Institute of Technology, Kornblumenstrasse 13, Karlsruhe, Germany

83 Edinburgh Genomics, The Ashworth Laboratories, The University of 9 Edinburgh, The King's Buildings, Edinburgh EH9 3JT, UK

104 Institute of Evolutionary Biology, The Ashworth Laboratories, The University 11 of Edinburgh, The King's Buildings, Edinburgh EH9 3JT, UK

12 Corresponding author: Emanuel Heitlinger emanuelheitlinger@gmail.com 


\section{Introduction}

14 The precipitous decline of stocks of the European eel, Anguilla anguilla, over

15 the last decades has spurred new research on these important fish (Dekker, 2003a, 2003b). While direct human influence such as overfishing and the destruction and damming of coastal habitats are undoubtedly the main reasons for the collapse of the eel population, the introduction of non-native pathogens may have contributed (Sures and Knopf, 2004).

The swim bladder nematode Anguillicola crassus was introduced from Asia to Europe early in the 1980s (Kirk, 2003; Neumann, 1985; Taraschewski et al., 1987). A. crassus is native to the Japanese eel Anguilla japonica, and has made a host jump to the European eel An. anguilla. A microsatellite study (Wielgoss et al., 2008), analysis of mitochondrial markers (Wielgoss et al. 2008, Laetsch et al. 2012) and historical reports (Koops and Hartmann, 1989) suggest that Taiwan was the most likely source of the founding population of the parasite, likely introduced by an import of live An. japonica eels to Northern Germany.

Adult $A$. crassus live inside the swim bladder of eels of the genus Anguilla. Female parasites shed eggs containing the L2 larval stage, which are released via the faeces into the water column. After hatching and ingestion by an intermediate host (copepods or ostracods; Moravec et al., 2005), L3 larval stages are infectious to the eel. When the eel host takes up infective $\mathrm{L} 3$, these migrate through the intestinal wall and the body cavity to the wall of the swim bladder, where they feed on tissue. After two additional moults ( $L 3$ to L4, and L4 to adult) sexually dimorphic adults enter the lumen of the swim bladder where they mate (De Chaleroy et al., 1990).

The parasite occurs at a higher prevalence in European eels than in Asian eels, and infects $A n$. anguilla at higher infection intensities than An. japonica. Importantly, the parasite is more pathogenic to the European than to its 
41 native Asian host. While An. japonica mounts an immune response that 42 eliminates many larvae, An. anguilla fails to mount such a response. The 43 antibody response is delayed and insufficient (Knopf, 2006; Knopf and Lucius, 44 2008) and parasite larvae are not encapsulated in An. anguilla exposed to $A$.

45 crassus (Heitlinger et al., 2009). A. crassus grows larger and produces more 46 embryonated eggs in An. anguilla hosts compared to An. japonica, both in the 47 wild (Münderle et al., 2006) and in laboratory experiments (Knopf and 48 Mahnke, 2004). The inadequate immune response of An. anguilla, creating a 49 more benign environment for the parasite, has been proposed to be the main reason for the altered dynamics of the host-parasite system (Knopf, 2006).

We are also interested in possible parasite contributions to these changed dynamics, and in particular in the possibility that the European eelparasitising $A$. crassus have been selected in or have adapted to their new hosts. Under this model, nematode genetics would also contribute to the changed host-parasite dynamic. A genetic component of the differences between European and Asian $A$. crassus was identified in a cross-infection experiment under common garden conditions (Weclawski et al., 2013). European strains of $A$. crassus were found to differ in life history traits from ones sourced from Taiwan, independent of the experimental host species. In particular European nematodes had an accelerated development compared to Asian nematodes (Weclawski et al., 2013). In the same experiment two European isolates from Poland were found to be diverged from those from Germany and Taiwan for morphological traits used to differenciate Anguillicola species (Weclawski et al., 2014).

65 We hypothesise that these genetic differences may result from allelic differences between the two nematode populations that either result in changed structures of key host-parasite interface effectors, or that these differences result in changes in expression of key genes involved in the interface. We assessed transcriptomic differences between age- and sex- 
71 infection design to disentangle differences in gene expression induced by 72 intrinsic genetic differences of the nematodes from the influence of the host 73 environment. The sequence data also allowed us to genotype the nematodes 74 and test for non-neutral evolutionary processes influencing phenotypic and 75 transcriptomic differences. 
Methods

\section{Experimental infection of eels}

78

79

80

81

82

83

L2 larvae used for the infection were collected from the swim bladders of wild yellow and silver eels from the River Rhine near Karlsruhe (49.0271N ; $8.3119 \mathrm{E})$ and from Lake Müggelsee near Berlin $(52.4372 \mathrm{~N} ; 13.6467 \mathrm{E})$ in Germany. Taiwanese larvae were obtained from eels from an aquaculture adjacent to Kao Ping River in south Taiwan (22.6418N; 120.4440E) and from a second aquaculture in Yunlin county $(23.7677 \mathrm{~N}, 120.2335 \mathrm{E})$, approximately $150 \mathrm{~km}$ further north on the west coast of Taiwan.

An. anguilla were obtained from a farm in Northern Germany (Albe, HarenRütenbrock; 52.8383N; 7.1095E). An. japonica were caught at the glass-eel stage in the estuary of Kao-ping River (22.5074N; $120.4220 \mathrm{E})$ and transferred to Germany. The absence of $A$. crassus before the experiment was confirmed in 8 Anguilla japonica and 4 Anguilla anguilla. After an acclimatisation period of 4 weeks (An. anguilla) or when they reached a size of $>35 \mathrm{~cm}$ ( $A n$. japonica) eels were infected using a stomach tube. During the infection period water temperature was held constant at $20^{\circ} \mathrm{C}$. Eels were kept in $160 \mathrm{~L}$ tanks in groups of 5-10 individuals and provided with fresh, oxygenated water through continuous circulation. Eels were fed every two days with commercial fish pellets (Dan-Ex 2848, Dana Feed A/S Ltd, Horsens, Denmark) ad libitum.

At 55 - $56 \mathrm{dpi}$, eels were euthanized and dissected. The swim bladder was opened and after determination of their sex under a binocular microscope (Semi 2000, Zeiss, Germany), adult A. crassus were immediately immersed in RNAlater (Quiagen, Hilden, Germany).

The experiment has been approved by the responsible authorities (Regierungspräsidium Karlsruhe approval no. 35-9185.81/G-120/06 and 359185.81/G-31/07).

\section{RNA extraction and preparation of sequencing libraries}


104 RNA was extracted from 12 individual female nematodes and for 12 pools of

105 from 1 to 5 male nematodes using the RNeasy kit (Quiagen, Hilden, 106 Germany) (see table 1). The paired-end TruSeq RNA sample preparation kit 107 (Illumina) was used to generate paired-end sequencing libraries with insert 108 sizes of roughly $270 \mathrm{bp}$ from polyA-selected RNA following the manufacturer's 109 instructions. Multiple indexed paired-end adapters were used to enable 110 multiplexing of the 24 different sequencing libraries in 3 pools of 8 samples 111 each. These three pools all contained one random replicate each for each

112 treatment combination ensuring complete statistical independence of 113 replicates. The pools were sequenced on an Illumina Genome Analyzer IIX 114 following the manufacturer's instructions. Raw data have been deposited in 115 ENA under the study accession number SRP010338.

\section{De novo assembly, protein prediction and annotation}

117 Trinity (version r2013-02-16) (Grabherr et al., 2011) was used to assemble 118 raw sequencing reads into contigs representing transcripts and genes. 119 Transdecoder (as supplied with Trinity) was used to predict protein coding 120 genes. Based on these predicted proteins we obtained domain annotations 121 using InterproScan (RC4) (Zdobnov and Apweiler, 2001) and sequence 122 similarity using BLAST (Altschul et al., 1997) against SwissProt. Gene 123 ontology (GO) terms were obtained either through association with domains 124 in InterproScan (considered higher quality) or through assignment according 125 to similarity (BLAST with a bitsore cutoff of 50, to increase annotation 126 coverage).

127 We used the R-package topGO to traverse the annotation-graph and analyse 128 each node in the annotation for over-representation of the associated term in 129 focal gene-sets compared to an appropriate universal gene-set with the 130 "classic" method and Fisher's exact test (F-test). To test over-representation 131 of Interpro domains we similarly used F-tests. The assembly contigs, read 
132 coverages and assignment to host, xenobiont and nematode groups, as well

133 as contig sets identified through their differential expression are available for

134 browsing in the online afterParty resource established for $A$. crassus at

135 http://anguillicola.nematod.es.

\section{Mapping, abundance estimation and normalisation}

137 All sequencing reads were mapped to the full Trinity assembly (including host 138 and other contaminant contigs) using Bowtie version 2.1.0 (Langmead and 139 Salzberg, 2012) and processed using RSEM (Li and Dewey, 2011) as indicated 140 in the downstream analysis instructions of Trinity.

141 Briefly, ambiguously-mapping reads were assigned to the most appropriate 142 transcript with RSEMs maximum likelihood method and a rounded counts 143 (summed over technical replicates) for both the transcript and gene level 144 were obtained. The final data used for all expression estimates was then 145 calculated as fragments per kilobase of feature (transcript) per million 146 fragments mapped (FPKM) based on trimmed mean of $M$ values (TMM) 147 normalisation (Robinson and Oshlack, 2010).

148 Genes and transcripts with less than 100 FPKM added over all samples were 149 disregarded in further analyses. At this point we also excluded genes and 150 transcripts of likely xenobiont (eukaryotic co-bionts of the nematode and fish, 151 and laboratory contaminants) or host (eel, by comparison to a previous eel 152 transcriptome (Coppe et al., 2010), and fish, from a taxonomic subset of the $153 \mathrm{NCBI} \mathrm{nr}$ protein database) origin. We removed transcripts if BLAST hits (e154 value cutoff 1e-5) against any of the fish or prokaryote databases were better 155 than those against a nematode subset of $\mathrm{nr}$.

\section{Analysis of expression data}

157 We used multi dimensional scaling (MDS), hierarchical clustering and k158 means clustering to analyse the structure in complete expression data set as 159 well as in male and female subsets. Based on these data we excluded two 
160 outlier samples ("AJ_T26F" and "AA_T42M"; The label is comprised of a two

161 letter code for the host species [AJ|AA], a one letter code for the population

$162[\mathrm{R} \mid \mathrm{T}]$, an arbitrary number and one letter for worm sex[F|M]).

163 The R-package edgeR (version 2.4.1) (Robinson et al., 2010) was used to 164 build negative binomial generalised linear models of expression. Models were 165 based on a negative binomial distribution and the dispersion parameter for 166 each transcript was approximated with a trend depending on the overall level 167 of expression. In the maximal fitted model expression was regressed on 168 nematode sex, host-species and parasite population, including all their 169 interactions. The full model thus contained terms $\mathrm{Si}+\mathrm{Hj}+\mathrm{Pk}+(\mathrm{SH}) \mathrm{ij}+$ 170 (SP)ik $+(\mathrm{HP}) \mathrm{jk}+(\mathrm{SHP}) \mathrm{ijk}+\varepsilon$, where $\varepsilon$ is the residual variance, $\mathrm{Si}$ is the 171 effect of the ith sex (male or female), $\mathrm{Hj}$ is the effect of the jth host species 172 (An. anguilla or An. japonica), Pk is the effect of the kth population (European 173 or Asian), (SH)ij is the sex-by-species interaction and similarly for the other 174 interactions.

175 The hierarchical nature of generalised linear models was respected 176 considering (removing) all interaction effects of a main-term (e.g. (SP)ik, 177 (SH)ij and (SHP)ijk) when analysing models for the significance of that term 178 (e.g. Si). Resulting $\mathrm{p}$ values were corrected for multiple testing using the 179 method of Benjamini and Hochberg and differential expression was inferred 180 at a false discovery rate (FDR) of $5 \%$ (adjusted p-value of 0.05 ).

181 Alternatively we built the corresponding partial models with only the male 182 and female subsets of the samples and estimated significance of host species 183 and nematode population factors as before.

184 Random forests as implemented in the R-package RandomForest were used 185 to additionally test for the ability to obtain a robust classifier separating host186 species or nematode populations (and the combination of these factors) in 187 decision trees on the gene expression data. We performed these tests on the 188 full dataset and on subsets containing significant genes for focal contrasts in 
the GLMs.

190

191 Samtools (version 0.1.18; mpileup) (Li et al., 2009) was used to call

193 the Bowtie mapping used before for gene expression analysis. SNPs were

194 filtered to have at least a phred-scaled quality of 30.

195 A matrix of genotypes was extracted for in which " 0 " coded homozygous 196 reference, " 1 " heterozygous and " 2 " homozygous for the alternate allele.

197 This matrix was read using the R-package adegenet (Jombart, 2008) and 198 transformed to the other R-object types as needed for different packages.

199 Heterozygosity was calculated for individual nematodes using the R-package 200 Rhh (Alho et al., 2010). In addition to the relative heterozygosity we 201 estimated internal relatedness (Amos et al., 2001), homozygosity by locus 202 (Aparicio et al., 2006) and standardised heterozygosity (Coltman et al., 203 1999). F-statistics were calculated using the R-package hierfstat (Goudet, 204 2005) implementing the method of Weir and Cockerham (Weir and 205 Cockerham, 1984) and Hardy-Weinberg-Equilibrium (HWE) for individual loci 206 (SNPs) within populations was tested using the permutation method of the 207 genetics package, as recommended for low sample sizes.

208 For multivariate analyses the genotype matrix was transposed to a distance 209 matrix and analysed using neighbour joining and maximum parsimony trees 210 with the R-package phangorn (Schliep, 2011). We then used principal 211 component analysis (PCA) from the R-package adegenet (Jombart, 2008) to

212 visualize the overall structure of the genotype data. The appropriate number

213 of population clusters was estimated using k-means clustering of the first five

214 principal components and analysis of the bayesian information criterion (BIC;

215 function find.clusters). Discriminant analysis of principal components was

216 then used to rank loci according to their contribution to the single remaining

217 discriminant function between the two resulting groups (European vs. Asian). 
218 We used a dn/ds threshold of 0.5 to assume positive selection. When whole 219 genes with stretches potentially under different selection regimes are 220 considered this has been suggested and used before (Swanson et al. 2004).

221 Kendall rank correlation tau tests were used to investigate correlations 222 between different SNP, genotyping and expression statistics.

\section{Results}

\section{A common garden experiment}

225 Populations of European $A$. crassus (sourced from the Rhine and Lake 226 Müggelsee, Berlin, Germany) were compared to Taiwanese nematodes 227 (sourced from two distinct aquaculture operations) were used to infect both 228 European An. Anguilla and Taiwanese An. japonica in a shared facility in a 229 cross-infection experiment (similar to that of Weclawski et al., 2013). Adult 230 nematodes were recovered, sexed and subjected to deep RNA-Seq analyses 231 in a carefully randomized design. Adult female nematodes were large enough 232 to be sampled individually, but RNA recovery from the smaller male 233 nematodes meant that some male samples were pools of a small number (up 234 to 5) of individuals taken from the same host eel. The RNA-Seq data were 235 mapped to a transcriptome assembly, and after elimination of host transcript 236 contamination, expression levels of nematode genes were compared 237 between host species, sexes and treatments. The RNA-Seq data were also 238 used to define and score single nucleotide polymorphisms (SNP) between the 239 nematodes, and these genotyping data were used to explore the population 240 genetics of the nematodes and their gene expression responses to infecting 241 different hosts. Details of our methods and analyses are given in the 242 Methods.

243 More nematodes are recovered from matching host-parasite combinations 
245 At the early time point of development (55 - 56 days post-infection (dpi))

246 chosen in our experiment we recovered more nematodes from the European

247 population of $A$. crassus in An. anguilla and more of the Taiwanese population

248 in the An. japonica. This was true for both adult sexes of the nematode, as

249 well as for L3 and L4 larval stages (Figure 1; $p<0.05$ for the interaction

250 effects of host species and parasite population in generalized linear models).

251 In geographically-matched host-parasite combinations a mean of 7.8 (Taiwan)

252 and 9.5 (Europe) of the 50 nematodes experimentally administrated were

253 recovered as adults. For the cross-matched combinations of host species and

254 parasite population, recovery of adult stage was on average only 1.4

255 (European nematode/An. japonica) and 4.4 (Taiwanese nematode/An.

256 anguilla).

\section{Transcriptome assembly and annotation}

258 We processed 12 individual female nematodes and 12 male nematode 259 samples (batches of 1-5 individuals from one host) for Illumina RNA 260 sequencing and obtained datasets of between $8.7 \times 10^{6}$ and $15.2 \times 10^{6}$ read 261 pairs from each of the samples (Table 1 ). These reads were assembled into 262 initial contigs representing 49,816 putative transcripts deriving from 33,173 263 transcript groups (or putative genes). These data have been made available 264 for analysis in an afterParty resource (Jones and Blaxter, 2013) at 265 http://anguillicola.nematod.es. These transcripts contain 60\% (6788 of 266 11372) of the previously deposited transcript reconstructions from a Roche 267454 RNA-Seq experiment but were on average longer (median length $608 \mathrm{nt}$ 268 vs. 466 nt), more complete ( $68 \%$ vs. $12 \%$ including translation start codons) 269 and covered a higher percentage of the proteome of the related nematode 270 Brugia malayi (64\% vs. $37 \%$ ).

271 By applying stringent quality filtering for coverage (eliminating sequences 272 reflecting only errors in the deep sequencing data) and taxonomic origin we 273 selected a high-confidence $A$. crassus transcriptome that included 6,047 274 genes with 8,106 transcripts. This subset of transcripts was longer (median 
275 length $1794 \mathrm{nt}$ ) than the whole dataset, equivalently complete with respect

276 to translation start codons $(68 \%$, as for the full set) and but had reduced

277 coverage of the $B$. malayi proteome (51\%). The majority of the raw sequence

278 reads mapped to this high quality subset of the transcriptome assembly. The

279 number of sequence reads analysed for expression and sequence

280 polymorphism ranged from $5.0 \times 10^{6}$ to $9.7 \times 10^{6}$ per sample (Table 1 ).

281 After conceptual translation $6,633(81.8 \%)$ of the transcripts were decorated 282 with annotations based on protein similarity to SwissProt and 6542 (80.1\%)

283 with annotations based on InterPro domain signatures. For 5284 (65.2\%) of 284 the transcripts, Gene Ontology (GO) annotations were obtained through 285 these domain signatures (Supplementary Data File 1).

286

287

288

289

290

291

292

293

294

295

296

297

298

299

300

301

302

Gene expression differentiates sexes but not experimental hosts and parasite population

Multi dimensional scaling (MDS) of the overall expression data robustly grouped male and female nematode samples but failed to separate samples from different experimental hosts or by nematode geographical origin (Supplementary Figure S1a). Similarly female and male nematode samples clustered distinctly in hierarchical clustering of the overall expression data. The same clustering failed to differentiate samples from European and Asian experimental host species or nematode geographical origin (Figure $1 \mathrm{~b}$ ). It was not possible to build a classifier grouping samples according to experimental host or parasite geographical population using random forests. The analysis prompted us, however, to exclude two samples ("A)_T26F" and "AA_T42M") from further expression analysis based on their overall outlier expression profiles.

We identified $2154(26.6 \%)$ of the transcripts as being significantly (FDR < 0.05; log fold-change $>1.5$ ) differentially expressed between female and male nematodes (Supplementary Data File 1) based on generalised linear 
303 models taking into account all analysed factors (nematode sex, experimental 304 host species and parasite population). The same models and thresholds 305 recovered only very small sets of genes differentially expressed between the 306 host species (27 transcripts; Supplementary Data File 2) and the parasite

307 populations (30 transcripts, Supplementary Data File 3). These small sets of 308 genes did not distinguish experimental host species or nematode populations 309 in hierarchical clustering (Figure 1 b and c) or MDS analysis (Supplementary 310 Figure S1 b and c). Random forests also failed to find robust classifiers based 311 on only these subsets of putatively differentially expressed genes.

312 Coding sequence polymorphism and positive selection

313 We identified panel of 128,707, bi-allelic, SNPs in 5008 genes over all 314 nematode samples. The overall ratio of transitions to transversions rates 315 (Ts:Tv) was 3.2. This can be expected in a transcriptome dataset due to a 316 higher ratio of transitions in coding regions. We determined the effect of

317 individual SNPs on coding sequence based on the conceptual translation and 318 found 46,815 synonymous and 27,326 non-synonymous substitutions. The 319 remaining 56,758 SNPs were in presumed untranslated regions (UTR), outside 320 of open reading frames. The 13.28 SNPs per 1000 sites comprised 27.36 321 synonymous SNPs per 1000 synonymous sites and 5.49 non-synonymous 322 SNPs per 1000 non-synonymous sites. This resulted in an overall ratio of 323 nonsynonymous substitutions per non-synonymous site over the synonymous 324 substitutions per synonymous site (dn/ds) of 0.20 .

325 Per-gene dn/ds was positively correlated with the total number of SNPS 326 detected in (Kendall rank correlation tau test $p<0.001$ ), so contigs with 327 fewer SNPs also had a lower $\mathrm{dn} / \mathrm{ds}$ on average. On the other hand the 328 number of SNPs per gene was found to be positively correlated with the 329 overall strength of gene expression (Kendall rank correlation tau tests $330 \mathrm{p}<0.001$ ). In contrast $\mathrm{dn} / \mathrm{ds}$ was negatively correlated (Kendall rank 
331 correlation tau tests $p<0.001$ ) with overall expression strength. Thus genes

332 with higher overall expression had more SNPs but lower dn/ds, even while in

333 general genes with more SNPs usually had a higher $\mathrm{dn} / \mathrm{ds}$.

334 Testing for GO term enrichment in the set of genes with high dn/ds ratios 335 ( $\mathrm{dn} / \mathrm{ds}>0.5)$ highlighted "zinc ion binding" and "protein binding" as over-

336 represented molecular functions, "regulation of apoptotic process" and 337 "cellular component biogenesis" as over-represented biological processes 338 and "intrinsic to endoplasmic reticulum membrane" and "intracellular 339 membrane-bounded organelle" as over represented cellular compartments 340 among genes under positive selection. We note that these terms are all high341 level GO terms and are thus relatively uninformative as to the shared 342 functions of the selected gene set. This gene-set putatively under positive 343 selection was not significantly enriched for signal sequences potentially 344 leading to secretion (Fisher's exact test $p=0.24$ ).

345 European and Asian nematode populations are genetically 346 differentiated

347 The European nematodes derived from an introduction, possibly of a small 348 population, from a source in Asia, and would be expected to be genetically 349 less diverse than the Asian nematodes, and nested within the diversity of the 350 Asian nematodes. Inbreeding depression in a population with restricted 351 diversity can result in an overabundance of homozygous genotypes. We 352 inferred genotype statistics for the individual nematodes assayed in our 353 experiment. We limited these heterozygosity based statistics to samples for 354 which only one individual nematode was sequenced, as for pooled 355 nematodes heterozygosity would obviously have been overestimated.

356 We detected no reduction of heterozygosity in European nematodes: neither 
357 relative heterozygosity (the ratio of heterozygous over homozygous 358 genotypes), internal relatedness (Amos et al., 2001), homozygosity by locus 359 (Aparicio et al., 2006) or standardised heterozygosity (Coltman et al., 1999)

360 from these data indicated significantly higher heterozygosity in the Asian 361 compared to the European nematodes (Table 2; one sided Mann-Whitney362 Wilcoxon tests, $p>0.05)$. The observed relative heterozygosity was for all 363 individuals higher than expected heterozygosity (0.173 in European, 0.175 in 364 Taiwanese samples). The overall inbreeding coefficient $F_{I S}$ (the correlation of 365 an individual's genotypes with genotypes found in European and Asian 366 subpopulations) was negative $(-0.0544)$, indicating that individual nematodes 367 are less related than expected from a model of random mating within their 368 population.

369 The overall fixation index $\left(F_{s t}\right)$ between European and Asian samples was 3700.045 , suggesting a rather low population structure. Nevertheless, a test for 371 differentiation using G-statistics (Goudet, 2005; Goudet et al., 1996), 372 indicated that it corresponds to highly significant genetic differentiation 373 between populations. No significant differentiation was found within the 374 European nematodes (between those sampled in the River Rhine and Berlin) 375 or within Taiwanese nematodes (between the different sampling sites) as far 376 as this could be analysed based on the low sample sizes for these 377 subpopulations.

378 Tests for Hardy-Weinberg-Equilibrium (HWE) within populations were only 379 possible for a subset (59\%) of SNP markers and HWE could only be rejected 380 ( $p<0.05$; in a permutation test) for 293 SNPs in the European population and 3815407 SNPs in the Taiwanese population.

382 Because allele frequency based calculations could only be performed for 383 nematodes that were sampled individually, we also used multivariate 384 statistical analysis, which does not strictly depend on inference of 


\section{PeerJ Reviewing Manuscript}

385 heterozygosity and can therefore be used to analyse non-individual 386 genotyping data (those missing from Table 2 ).

387 Population differentiation between nematodes sampled in Europe and Taiwan 388 was also pronounced in this multivariate analysis as the distances between 389 genotype matrices revealed a separation of genotypes from European and 390 the Taiwanese populations. This differentiation was visible in both neighbour391 joining and maximum parsimony trees computed on the distance matrix 392 (Figure $3 \mathrm{a}$ and $\mathrm{b}$ ).

393 Further validation was provided by principal component analysis, in which the 394 first component (explaining 12\% of the total variance) separated nematodes 395 from Taiwan and Europe clearly (Figure $3 \mathrm{c}$ ). The second principal component 396 (explaining 9\% of total variance) differentiated some of the European 397 nematodes but did not show a clear pattern regarding origin (within Europe) 398 or any other characteristics of the sampled nematodes.

399 More synonymous polymorphism are found in genotypes 400 distinguishing between populations

401 Clustering analysis (k-means) of principal components revealed a structure of 402 only two clearly distinguishable groups in the data, identical to the European 403 and Taiwanese samples. This was further validated by discriminant analysis 404 of principal components (DAPC). The discriminant function (largely similar to 405 principal component 1) permitted $100 \%$ accurate assignment of individual 406 nematodes in bootstrapping tests to the correct source population, again 407 demonstrating a clear differentiation between the European and Taiwanese 408 samples (Figure $4 \mathrm{a}$ and $\mathrm{b}$ ).

409 Genes associated with differentiation between nematode populations 410 (maximal DAPC variable contribution $>0.8 \times 10^{-5}$ of a locus in the gene), were 411 enriched for the GO terms "receptor signaling protein" and "exopeptidase" 412 activity (molecular function), "endoplasmic reticulum membrane" and signal 413 "peptidase complex" (cellular compartment). These genes were also 
414 significantly enriched for signal sequences leading potentially to secretion

415 (Fishers exact test $p<0.001$ ).

416 The variable contribution of individual alleles to the discriminant function

417 from DAPC was strongly correlated with $F_{\text {st }}$ (Supplementary Figure S2 a), 418 highlighting the agreement of both analyses in sorting loci for their 419 contributing to the differentiation between the two nematode populations.

420 Loci with a stronger contribution to the differentiation between European and 421 Taiwanese nematodes showed an overrepresentation of synonymous SNPs 422 and SNPs in UTRs (Figure S2 b). All measures of differentiation used ( $F_{s t}, F_{\text {is }}$, 423 contribution to the discriminant function and PC1 loading) were thus 424 significantly higher for polymorphisms without an effect on the protein 425 sequence (Mann-Whitney-Wilcoxon tests, $p<0.001$ ).

426 Differential gene expression between sexes is negatively correlated with genotypic differentiation between populations

428 Both the mean and maximal signals for genotypic separation in populations 429 over loci per gene ( $F_{\text {st }}$, contribution to discriminant function, loading of PC1) 430 were negatively correlated with absolute values of log fold-change between 431 male and females. Similarly the p-values for expression differences between 432 sexes were positively correlated with all measurements of genotypic 433 differentiation. Thus genes expressed more differentially between sexes were 434 associated with lower genotypic differentiation between populations. This is 435 especially striking as higher expression was not associated with genotypic 436 differentiation overall. 


\section{Discussion}

438 Common garden experiments are classical method in evolutionary biology to

439 disentangle genetic from environmentally induced effects. A cross infection

440 experiment is the obvious extension of this approach to a host-parasite 441 system.

442 We used a common garden experiment to demonstrate that $A$. crassus shows 443 an "adapted" ability to successfully infect An. anguilla and An. japonica, as 444 more parasites were recovered in matching infections (European parasites in 445 An. anguilla; Taiwanese parasites in An. japonica). These differences are in 446 good agreement with previous data at an earlier time of infection in a similar 447 experiment (Weclawski et al., 2013). As noted before, this ("adapted") 448 pattern does not necessarily reflect adaptation, as we can not assume that 449 earlier development leads to higher parasite fitness, which would better be 450 measured by lifetime reproductive success.

451 We analysed parasite geographical source and host environment induced 452 differences on gene-expression using transcriptomics. We did not detect 453 differences in gene expression between the parasites infecting An. japonica 454 and An. anguilla. The gene expression profiles of young adult stages of $A$. 455 crassus seem inert to the environment imposed by the different host species. 456 This is unexpected, as large morphological differences are observed between 457 nematodes from different host species in the wild (Münderle et al., 2006) and 458 in laboratory infections (Knopf and Mahnke, 2004; Weclawski et al., 2013; 459 Weclawski et al., 2014). A possible reason might be that nematodes are 460 influenced by and respond to the host immune system only during the larval 461 stages migrating through tissues. The transcriptome of haematophagous 462 adult stages living in the swim bladder may be rather unaffected by the host 463 environment, and the phenotypic responses (parasite size) a result of 464 improved larval health in the compromised host. It is possible that our 465 experimental design might have selected nematodes with a "healthy" 466 transcriptome, one that allows survival irrespective of the experimental host. 


\section{PeerJ Reviewing Manuscript}

467 This "healthy" transcriptome might then be largely the same in different host 468 environments.

469 We identified no transcriptomic signature of the differences in life history 470 traits between European and Taiwanese nematode populations that have 471 been reported from cross-infection experiments irrespective of the host 472 environment (Weclawski et al., 2013). The faster development of the 473 European population of A. crassus thus has no obvious correlation with an 474 early adult expression phenotype.

475 A previous report of tentative gene expression differences between single 476 European and Taiwanese nematodes in their respective natural hosts 477 (Heitlinger et al., 2013) may be explained as a product of stochastic noise in 478 transcriptome sampling without repetition. The sampling size used for 479 present study (24 nematodes, six per treatment group) is relatively high for a 480 transcriptomics study and, while it is never possible to prove a negative, we 481 consider the present negative results to be true negatives.

482 As an argument supporting this notion, our analysis was able to find sex 483 dimorphic expression differences in roughly one third of the analysed genes. 484 This difference corresponds to a good resolution according to roughly one to 485 two third of genes showing differences between sexes throughout many 486 animal taxa (including studies with even higher repetition) (Cutter and Ward, 487

As high as it is for a transcriptomic experiment, the sample size of 24 nematodes from two populations is very small for a population study. Nevertheless, we demonstrate that a combination of classical and multivariate analysis can be useful for population genetic screening of such a dataset originally obtained for analysing gene expression. High-density genotyping of nematodes from our transcriptome sequencing demonstrated that the European parasite population is not a genetic sub-sample of the 
496 from Europe and Taiwan, but not within sampling sites on the Taiwanese East

497 cost and from Southern and North-Eastern Germany indicates that the 498 European population of $A$. crassus might have a genetically distinct origin 499 from our Taiwanese isolates.

500 The coefficient of population differentiation $F_{\text {st }}$ (Wright, 1949) between 501 European and Taiwanese individuals had a value of 0.045 and could be 502 interpreted as indicating negligible differentiation. Nevertheless, we show 503 that, based on tests using g-statistics and multivariate analysis, the deep set 504 of SNPs is fully sufficient to assign nematodes to European or Asian 505 populations without any error. These pairwise $F_{\text {st }}$ values between Taiwanese 506 and European samples are in line with previous findings of Wielgoss et al. 507 (2008), who observed values between 0.02 and 0.056 .

508 DAPC permitted the measurement of the degree of differentiation for both 509 genes and samples. The contribution of genes to the discriminant function is 510 highly correlated with $F_{\text {st, }}$ but has advantages for low sampling sizes and 511 ultra-deep sampling of markers throughout the genome (Jombart et al., 512 2010).

513 We can also conclude from low values of the overall inbreeding coefficient $F_{\text {is }}$ 514 that the European populations of $A$. crassus show no heavy signature of 515 inbreeding after a genetic bottleneck. Additional evidence for this is provided 516 by high values of heterozygosity for individual European nematodes, which 517 are not reduced compared to Taiwanese individuals. This result is largely in 518 line with a previous study, which reported an universally high heterozygosity 519 in Northern Europe, using larger sample sizes (Wielgoss et al., 2008). The 520 same authors observed only marginally higher heterozygosity in isolates from 521 Taiwan.

522 In the present study, however, we did not analyse Taiwanese nematodes from 523 free-living eels. Individual $A$. crassus from wild caught eels displayed higher 524 heterozygosity than those sampled from aquaculture operations in our own 
525 studies before (Heitlinger et al., 2013) and thus some additional gentoypic

526 diversity is likely to be found within Taiwan.

527

528 Populations from Taiwanese aquacultures might not be in Hardy-Weinberg

529 Equillibrium, as this might only fail to be rejected in most markers due to the

530 low sample sizes. It cannot be excluded that Taiwanese isolates are from

531 populations experiencing a strong Wahlund effect, as observed in isolates

532 from the River Rhine (Wielgoss et al., 2010). A continued mixing of population

533 could have resulted in the affiliation of our two different Taiwanese over the

534 European isolates.

535 In the absence of gene flow within approximately 100 generations since its 536 introduction, $A$. crassus populations could have undergone substantial 537 genetic drift, explaining the clear distinction from the presumed Taiwanese 538 source populations. Alternatively, the Taiwanese populations may not be so 539 closely related to the actual source of European $A$. crassus, and Asian 540 populations of $A$. crassus more closely related to the true source population 541 of the European isolates may be identified in future studies involving larger 542 sample sizes. In the meantime we encourage caution in discussion of genetic 543 differences in European and Taiwanese isolates of $A$. crassus as having been 544 induced by their translocation (i.e. selection by host or environment), as the 545 same differences may already be present between unsampled Asian isolates.

546 For our present study the patterns of SNPs in protein-coding genes of $A$. 547 crassus is informative. The estimated $d n / d s$ of 0.2 is only slightly lower than 548 the 0.244 previously obtained from 454 pyrosequencing of the transcriptome 549 (Heitlinger et al., 2013). As expected, genes with overall higher gene 550 expression had a lower $\mathrm{dn} / \mathrm{ds}$, probably because genes with higher 551 expression are under stronger purifying selection (Drummond et al., 2005). 
552 Our previous analyses identified proteinases as possibly being under positive 553 selection (Heitlinger et al., 2013), but we did not observe enrichment of for 554 proteinases in the set of putatively positively selected genes (using the same 555 threshold of $\mathrm{dn} / \mathrm{ds}>0.5$ ). The reason for this are mainly additional non556 coding SNPs observed in the respective proteinase genes (see below why 557 some peptidases still show an interesting pattern of genotypes).

558 Merging population differentiation estimates with information on coding 559 polymorphism we found that loci contributing to the differences between 560 European and Taiwanese $A$. crassus were enriched for synonymous 561 polymorphisms. These loci therefore do not show evidence of positive 562 selection. Nevertheless genes associated with loci that discriminated 563 between populations showed enrichment for functional categories that might 564 be important for host-parasite interaction, and variation in these genes could 565 explain differences between Taiwanese and European A. crassus.

566 Signal sequences that direct newly translated proteins to enter the secretory 567 system of the endoplasmic reticulum were found more often than expected in 568 genes that differentiated between populations. Additionally signal peptidase569 associated processing at the endoplasmic reticulum membrane and general 570 endopeptidases showed high differentiation between $A$. crassus from Taiwan 571 and Europe. Interestingly three of these endopeptidases were among the 572 twelve peptidases reported previously to possess a high $\mathrm{dn} / \mathrm{ds}$ and to be 573 potentially under positive selection (Heitlinger et al., 2013).

574 While the present study does not find an excessively high $\mathrm{dn} / \mathrm{ds}$ in these 575 peptidases, these data are suggestive of a key role of secreted peptides and 576 their processing in the differences between $A$. crassus from Europe and 577 Taiwan. 


\section{Acknowledgments}

579 We thank Yun-San Han and his group at National Taiwan University for 580 support obtaining Japanese Eels and $A$. crassus larvae. We thank the staff of 581 Edinburgh Genomics for processing samples. We thank Sebastien Weigloss 582 for comments on the manuscript and analyses. 


\section{References}

Alho, J.S., Välimäki, K., Merilä, J., 2010. Rhh: an R extension for estimating multilocus heterozygosity and heterozygosity-heterozygosity correlation. Mol. Ecol. Resour. 10, 720-722. doi:10.1111/j.1755-0998.2010.02830.x

Altschul, S.F., Madden, T.L., Schäffer, A.A., Zhang, J., Zhang, Z., Miller, W., Lipman, D.J., 1997. Gapped BLAST and PSI-BLAST: a new generation of protein database search programs. Nucleic Acids Res. 25, 3389-3402. doi:10.1093/nar/25.17.3389

Amos, W., Wilmer, J.W., Fullard, K., Burg, T.M., Croxall, J.P., Bloch, D., Coulson, T., 2001. The influence of parental relatedness on reproductive success. Proc. Biol. Sci. 268, 2021-2027. doi:10.1098/rspb.2001.1751

Aparicio, J.M., Ortego, J., Cordero, P.J., 2006. What should we weigh to estimate heterozygosity, alleles or loci? Mol. Ecol. 15, 4659-4665. doi:10.1111/j.1365294X.2006.03111.x

Coltman, D.W., Pilkington, J.G., Smith, J.A., Pemberton, J.M., 1999. Parasite-Mediated Selection against Inbred Soay Sheep in a Free-Living, Island Population. Evolution 53, 1259. doi: $10.2307 / 2640828$

Coppe, A., Pujolar, J.M., Maes, G.E., Larsen, P.F., Hansen, M.M., Bernatchez, L., Zane, L., Bortoluzzi, S., 2010. Sequencing, de novo annotation and analysis of the first Anguilla anguilla transcriptome: EeelBase opens new perspectives for the study of the critically endangered european eel. BMC Genomics 11, 635. doi:10.1186/1471-2164-11-635

Cutter, A.D., Ward, S., 2005. Sexual and temporal dynamics of molecular evolution in C. elegans development. Mol. Biol. Evol. 22, 178-188. doi:10.1093/molbev/msh267

De Chaleroy, D., Grisez, L., Thomas, K., Belpaire, C., Ollevier, F., 1990. The life cycle of Anguillicola crassus. Dis. Aquat. Organ. 8, 77-84.

Dekker, W., 2003a. Status of the European eel stock and fisheries. Eel Biol. 237-254.

Dekker, W., 2003b. Worldwide decline of eel resources necessitates immediate action. Québec Declaration of Concern. Fisheries 12, 28-30.

Drummond, D.A., Bloom, J.D., Adami, C., Wilke, C.O., Arnold, F.H., 2005. Why highly expressed proteins evolve slowly. Proc. Natl. Acad. Sci. U. S. A. 102, 1433814343.

Goudet, J., 2005. Hierfstat, a package for R to compute and test hierarchical F-statistics. Mol. Ecol. Notes 5, 184-186.

Goudet, J., Raymond, M., de Meeüs, T., Rousset, F., 1996. Testing differentiation in diploid populations. Genetics 144, 1933-1940. 
Grabherr, M.G., Haas, B.J., Yassour, M., Levin, J.Z., Thompson, D.A., Amit, I., Adiconis, X., Fan, L., Raychowdhury, R., Zeng, Q., Chen, Z., Mauceli, E., Hacohen, N., Gnirke, A., Rhind, N., di Palma, F., Birren, B.W., Nusbaum, C., Lindblad-Toh, K., Friedman, N., Regev, A., 2011. Full-length transcriptome assembly from RNA-Seq data without a reference genome. Nat. Biotechnol. 29, 644-652. doi:10.1038/nbt.1883

Heitlinger, E., Bridgett, S., Montazam, A., Taraschewski, H., Blaxter, M., 2013. The transcriptome of the invasive eel swimbladder nematode parasite Anguillicola crassus. BMC Genomics 14, 87. doi:10.1186/1471-2164-14-87

Heitlinger, E.G., Laetsch, D.R., Weclawski, U., Han, Y.-S., Taraschewski, H., 2009. Massive encapsulation of larval Anguillicoloides crassus in the intestinal wall of Japanese eels. Parasit. Vectors 2, 48. doi:10.1186/1756-3305-2-48

Jin, W., Riley, R.M., Wolfinger, R.D., White, K.P., Passador-Gurgel, G., Gibson, G., 2001. The contributions of sex, genotype and age to transcriptional variance in Drosophila melanogaster. Nat. Genet. 29, 389-395. doi:10.1038/ng766

Jombart, T., 2008. adegenet: a $\mathrm{R}$ package for the multivariate analysis of genetic markers. Bioinformatics 24, 1403-1405.

Jombart, T., Devillard, S., Balloux, F., 2010. Discriminant analysis of principal components: a new method for the analysis of genetically structured populations. BMC Genet. 11, 94. doi:10.1186/1471-2156-11-94

Jones, M., Blaxter, M., 2013. afterParty: turning raw transcriptomes into permanent resources. BMC Bioinformatics 14, 301. doi:10.1186/1471-2105-14-301

Kirk, R.S., 2003. The impact of Anguillicola crassus on European eels. Fish. Manag. Ecol. 10, 385-394. doi:10.1111/j.1365-2400.2003.00355.x

Knopf, K., 2006. The swimbladder nematode Anguillicola crassus in the European eel Anguilla anguilla and the Japanese eel Anguilla japonica: differences in susceptibility and immunity between a recently colonized host and the original host. J. Helminthol. 80, 129-36. doi:16768856

Knopf, K., Lucius, R., 2008. Vaccination of eels (Anguilla japonica and Anguilla anguilla) against Anguillicola crassus with irradiated L3. Parasitology 135, 633-40. doi:S0031182008004162

Knopf, K., Mahnke, M., 2004. Differences in susceptibility of the European eel (Anguilla anguilla) and the Japanese eel (Anguilla japonica) to the swim-bladder nematode Anguillicola crassus. Parasitology 129, 491-6. doi:15521638

Koops, H., Hartmann, F., 1989. Anguillicola-infestations in Germany and in German eel imports. J. Appl. Ichthyol. 5, 41-45.

Laetsch, D., Heitlinger, E., Taraschewski, H. Nadler, S., Blaxter, M., (2012) The phylogenetics of Anguillicolidae (Nematoda: Anguillicoloidea), swimbladder parasites of eels. BMC evolutionary biology 12:60. doi:10.1186/1471-2148-12-60.

Langmead, B., Salzberg, S.L., 2012. Fast gapped-read alignment with Bowtie 2. Nat. Methods 9, 357-359. doi:10.1038/nmeth.1923 
Li, B., Dewey, C., 2011. RSEM: accurate transcript quantification from RNA-Seq data with or without a reference genome. BMC Bioinformatics 12, 323.

Li, H., Handsaker, B., Wysoker, A., Fennell, T., Ruan, J., Homer, N., Marth, G., Abecasis, G., Durbin, R., 2009. The sequence alignment/map format and SAMtools. Bioinformatics 25, 2078-2079.

Münderle, M., Taraschewski, H., Klar, B., Chang, C.W., Shiao, J.C., Shen, K.N., He, J.T., Lin, S.H., Tzeng, W.N., 2006. Occurrence of Anguillicola crassus (Nematoda: Dracunculoidea) in Japanese eels Anguilla japonica from a river and an aquaculture unit in SW Taiwan. Dis. Aquat. Organ. 71, 101-8. doi:16956057

Moravec, F., Nagasawa, K., Miyakawa, M., 2005. First record of ostracods as natural intermediate hosts of Anguillicola crassus, a pathogenic swimbladder parasite of eels Anguilla spp. Dis. Aquat. Organ. 66, 171-3. doi:16231644

Neumann, W., 1985. Schwimmblasenparasit Anguillicola bei Aalen. Fisch. Teichwirt Fachz. Für Binnenfisch. 11.

Robinson, M.D., McCarthy, D.J., Smyth, G.K., 2010. edgeR: a Bioconductor package for differential expression analysis of digital gene expression data. Bioinformatics 26 , 139-140.

Robinson, M.D., Oshlack, A., 2010. A scaling normalization method for differential expression analysis of RNA-seq data. Genome Biol. 11, R25. doi:10.1186/gb2010-11-3-r25

Schliep, K.P., 2011. phangorn: Phylogenetic analysis in R. Bioinformatics 27, 592-593.

Sures, B., Knopf, K., 2004. Parasites as a Threat to Freshwater Eels? Science 304, 209-211. doi:10.1126/science.304.5668.209

Swanson W.J., Clark A.G., Waldrip-Dail H.M., Wolfner M.F., Aquadro C.F., 2001. Evolutionary EST analysis identifies rapidly evolving male reproductive proteins in Drosophila. Proc Natl Acad Sci USA, 98:7375-7379.

Taraschewski, H., MORAVEC, F., LAMAH, T., ANDERS, K., 1987. Distribution and morphology of two helminths recently introduced into European eel populations: Anguillicola crassus(Nematoda, Dracunculoidea) and Paratenuisentis ambiguus(Acanthocephala, Tenuisentidae). Dis. Aquat. Organ. 3, 167-176.

Weclawski, U., Heitlinger, E.G., Baust, T., Klar, B., Petney, T., San Han, Y., Taraschewski, H., 2013. Evolutionary divergence of the swim bladder nematode Anguillicola crassus after colonization of a novel host, Anguilla anguilla. BMC Evol. Biol. 13, 78.

Weclawski, U., Heitlinger, E.G., Baust, T., Klar, B., Petney, T., San Han, Y., Taraschewski, H., 2014. Rapid evolution of Anguillicola crassus in Europe: species diagnostic traits are plastic and evolutionarily labile. Front. Zool., 11, 74.

Weir, B.S., Cockerham, C.C., 1984. Estimating F-statistics for the analysis of population structure. evolution 1358-1370.

Wielgoss, S., Hollandt, F., Wirth, T., Meyer, A., 2010. Genetic signatures in an invasive parasite of Anguilla anguilla correlate with differential stock management. J. Fish 
Biol. 77, 191-210. doi:10.1111/j.1095-8649.2010.02670.x

Wielgoss, S., Taraschewski, H., Meyer, A., Wirth, T., 2008. Population structure of the parasitic nematode Anguillicola crassus, an invader of declining North Atlantic eel stocks. Mol. Ecol. 17, 3478-95. doi:MEC3855

Wright, S., 1949. The genetical structure of populations. Ann. Eugen. 15, 323-354.

Yang, X., Schadt, E.E., Wang, S., Wang, H., Arnold, A.P., Ingram-Drake, L., Drake, T.A., Lusis, A.J., 2006. Tissue-specific expression and regulation of sexually dimorphic genes in mice. Genome Res. 16, 995-1004. doi:10.1101/gr.5217506

Zdobnov, E.M., Apweiler, R., 2001. InterProScan - an integration platform for the signature-recognition methods in InterPro. Bioinformatics 17, 847-848. doi:10.1093/bioinformatics/17.9.847 


\section{Table 1 (on next page)}

Samples analysed for transcriptome response 


\section{PeerJ Reviewing Manuscript}

\begin{tabular}{|c|c|c|c|c|c|c|c|}
\hline sample name & $\begin{array}{l}\text { experimenal } \\
\text { host species }\end{array}$ & worm sex & worm population & $\begin{array}{l}\text { \# worms } \\
\text { prepared }\end{array}$ & raw reads & mapped reads & analysed reads \\
\hline AA_R11M & An. anguilla & male & Europe (Rhine) & 14 & 11986442 & 8783231 & 761996 \\
\hline AA_R16M & An. anguilla & male & Europe (Rhine) & 4 & 10810349 & 7437741 & 615026 \\
\hline AA_R18F & An. anguilla & female & Europe (Rhine) & 1 & 9227615 & 6720900 & 5428268 \\
\hline AA_R28F & An. anguilla & female & Europe (Rhine) & 1 & 10135670 & 7044401 & 559233 \\
\hline AA_R2M & An. anguilla & male & Europe (Berlin) & 4 & 12469746 & 8745921 & 740808 \\
\hline AA_R8F & An. anguilla & female & Europe (Berlin) & 1 & 15270570 & 11371346 & 968705 \\
\hline AA_T12F & An. anguilla & female & Taiwan (KaoPing) & 1 & 11299438 & 8196168 & 6727218 \\
\hline AA_T20F & An. anguilla & female & Taiwan (KaoPing) & 1 & 11740839 & 8575826 & 699427 \\
\hline AA_T24M & An. anguilla & male & Taiwan (KaoPing) & 3 & 8552723 & 6023322 & 5053565 \\
\hline AA_T3M & An. anguilla & male & Taiwan (Yulin) & 4 & 11031751 & 7783403 & 6730362 \\
\hline AA_T42M & An. anguilla & male & Taiwan (Yulin) & 1 & 11573501 & 8013752 & 6829319 \\
\hline AA_T45F & An. anguilla & female & Taiwan (Yulin) & 1 & 10646847 & 7554730 & 6314234 \\
\hline AJ_R1F & An. japonica & female & Europe (Rhine) & 1 & 9855005 & 6983544 & 5814315 \\
\hline AJ_R1M & An. japonica & male & Europe (Rhine) & 1 & 10211903 & 6951868 & 5828185 \\
\hline AJ_R3F & An. japonica & female & Europe (Rhine) & 1 & 9897937 & 7100162 & 5618547 \\
\hline AJ_R3M & An. japonica & male & Europe (Rhine) & 2 & 8775211 & 5981163 & 5006069 \\
\hline AJ_R5F & An. japonica & female & Europe (Berlin) & 1 & 11949105 & 8814614 & 7562071 \\
\hline AJ_R5M & An. japonica & male & Europe (Berlin) & 1 & 11231532 & 7859814 & 6651999 \\
\hline AJ_T19M & An. japonica & male & Taiwan (Yulin) & 7 & 9195576 & 6605467 & 5733247 \\
\hline AJ_T20M & An. japonica & male & Taiwan (Yulin) & 8 & 10862591 & 7715619 & 6437571 \\
\hline AJ_T25M & An. japonica & male & Taiwan (Yulin) & 5 & 11195315 & 7565845 & 6416480 \\
\hline AJ_T26F & An. japonica & female & Taiwan (Yulin) & 1 & 11195335 & 8051694 & 6833011 \\
\hline AJ_T5F & An. japonica & female & Taiwan (KaoPing) & 1 & 10357569 & 7415162 & 6152064 \\
\hline AJ_T8F & An. japonica & female & Taiwan (Yulin) & 1 & 14196382 & 10547153 & 866784 \\
\hline
\end{tabular}




\section{Table 2 (on next page)}

Heterozygosity measures for individual worms 
PeerJ Reviewing Manuscript

\begin{tabular}{|l|r|r|r|r|r|r|r|}
\hline \multicolumn{1}{|c|}{ Sample } & $\begin{array}{c}\text { homozygous } \\
\text { reference }\end{array}$ & heterozygous & $\begin{array}{c}\text { homozygous } \\
\text { alternate allele }\end{array}$ & $\begin{array}{c}\text { relative } \\
\text { heterozygosity }\end{array}$ & $\begin{array}{c}\text { internal } \\
\text { relatedness }\end{array}$ & $\begin{array}{c}\text { homozygosity } \\
\text { by locus }\end{array}$ & $\begin{array}{c}\text { statndardized } \\
\text { heterozygosity }\end{array}$ \\
\hline AA_R18F & 99890 & 25148 & 3669 & 0.24 & -0.45 & 0.15 & 1.01 \\
\hline AA_R28F & 98624 & 26049 & 4034 & 0.25 & -0.42 & 0.16 & 1.00 \\
\hline AA_R8F & 97935 & 26898 & 3874 & 0.26 & -0.40 & 0.16 & 0.99 \\
\hline AJ_R1F & 99075 & 25247 & 4385 & 0.24 & -0.43 & 0.16 & 1.00 \\
\hline AJ_R1M & 99646 & 24433 & 4628 & 0.23 & -0.43 & 0.15 & 1.01 \\
\hline AJ_R3F & 96540 & 28493 & 3674 & 0.28 & -0.40 & 0.17 & 0.98 \\
\hline AJ_R5F & 99080 & 25312 & 4315 & 0.24 & -0.44 & 0.15 & 1.01 \\
\hline AJ_R5M & 97330 & 27798 & 3579 & 0.28 & -0.40 & 0.17 & 0.99 \\
\hline AA_T12F & 97278 & 27141 & 4288 & 0.27 & -0.38 & 0.17 & 0.99 \\
\hline AA_T20F & 98479 & 27379 & 2849 & 0.27 & -0.41 & 0.16 & 1.00 \\
\hline AA_T42M & 99514 & 23700 & 5493 & 0.23 & -0.41 & 0.15 & 1.01 \\
\hline AA_T45F & 96282 & 28686 & 3739 & 0.29 & -0.38 & 0.17 & 0.98 \\
\hline AJ_T26F & 102425 & 22937 & 3345 & 0.22 & -0.46 & 0.14 & 1.03 \\
\hline AJ_T5F & 99387 & 24810 & 4510 & 0.24 & -0.42 & 0.15 & 1.01 \\
\hline AJ_T8F & 97539 & 26640 & 4528 & 0.26 & -0.39 & 0.16 & 0.99 \\
\hline
\end{tabular}




\section{Figure 1}

Recovery of nematode populations in experimental host species

An. anguilla and An. japonica were infected with a dose of 50 L2 stage larvae of Anguillcola crassus from European and Taiwanese populations. After 55 - 56 days different lifecycle stages of the nematodes were recovered and counted. Shown are the mean numbers of nematodes recovered from different host-parasite combinations, with errors bars indicating the standard error of these means. In host-parasite combinations occurring in the wild (European/European and Taiwan/Taiwan) more nematodes are recovered.

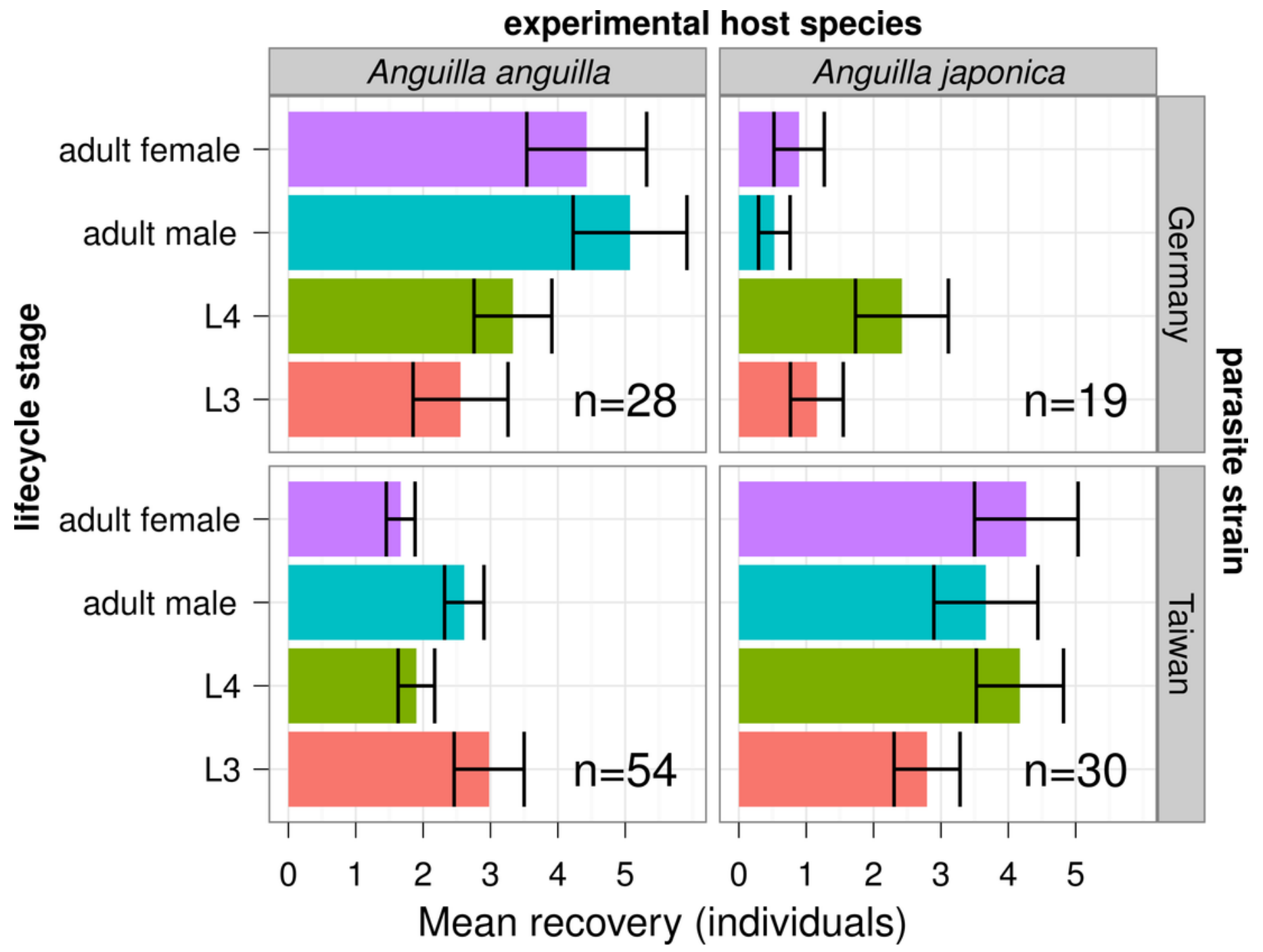




\section{Figure 2}

Overall gene expression differences according to nematode population and experimental host species

Gene expression changes in reconstructed transcripts were measured by mapping of sequencing reads. Normalized values were expressed in kilobase of feature (transcript) per million fragments mapped (FPKM) and plotted using the R-package pheatmap. Both rows (transcripts) and columns (nematode samples) were hierarchically clustered based on complete clustering of Euclidean distances. Panel A depicts all 8,106 transcripts. In this analysis, the nematode samples are grouped correctly according to their sex. Panel B shows the subset of 30 genes significantly differently expressed ( $F D R<0.05 ; \log F C>1.5$ ) between European and Asian nematode populations, and Panel $C$ the subset of 27 genes significantly differently expressed in the different experimental host species. Note that samples AJ_T26F and AA_T42M were removed because of their outlier status in the generalized linear models constructed for significance testing (with edgeR). They are thus also missing in Panels B and $\mathrm{C}$ and all other gene expression statistics. While overall gene expression clearly distinguishes male and female nematodes, even the putatively differentially expressed gene-sets for nematode population or experimental host species cannot distinguish their respective contrasts. 
a

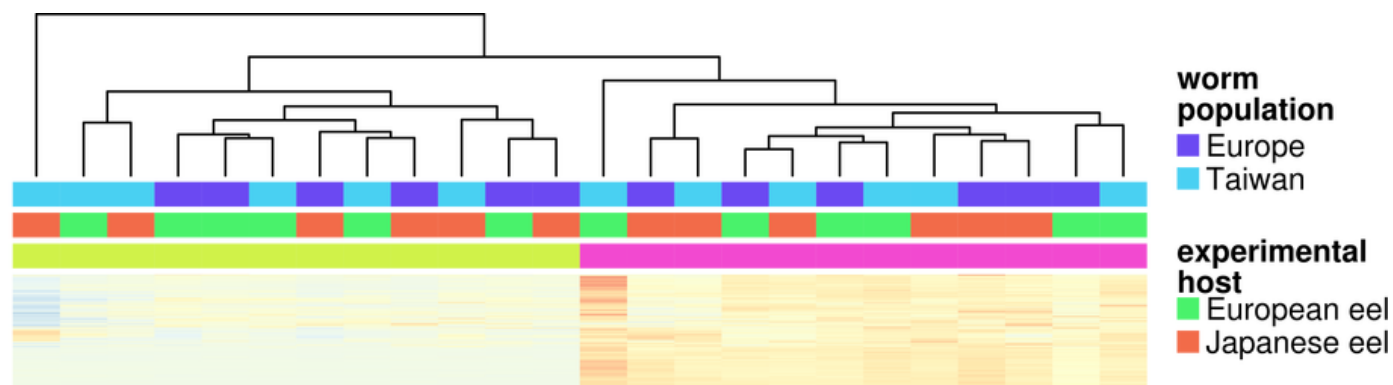

worm sex

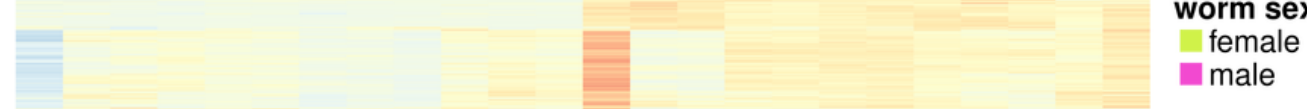

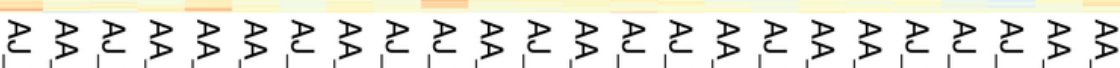

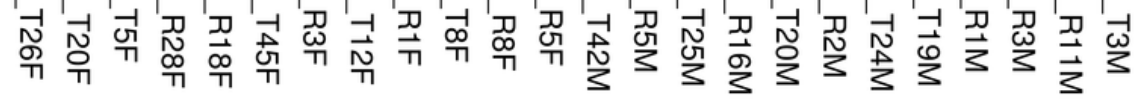
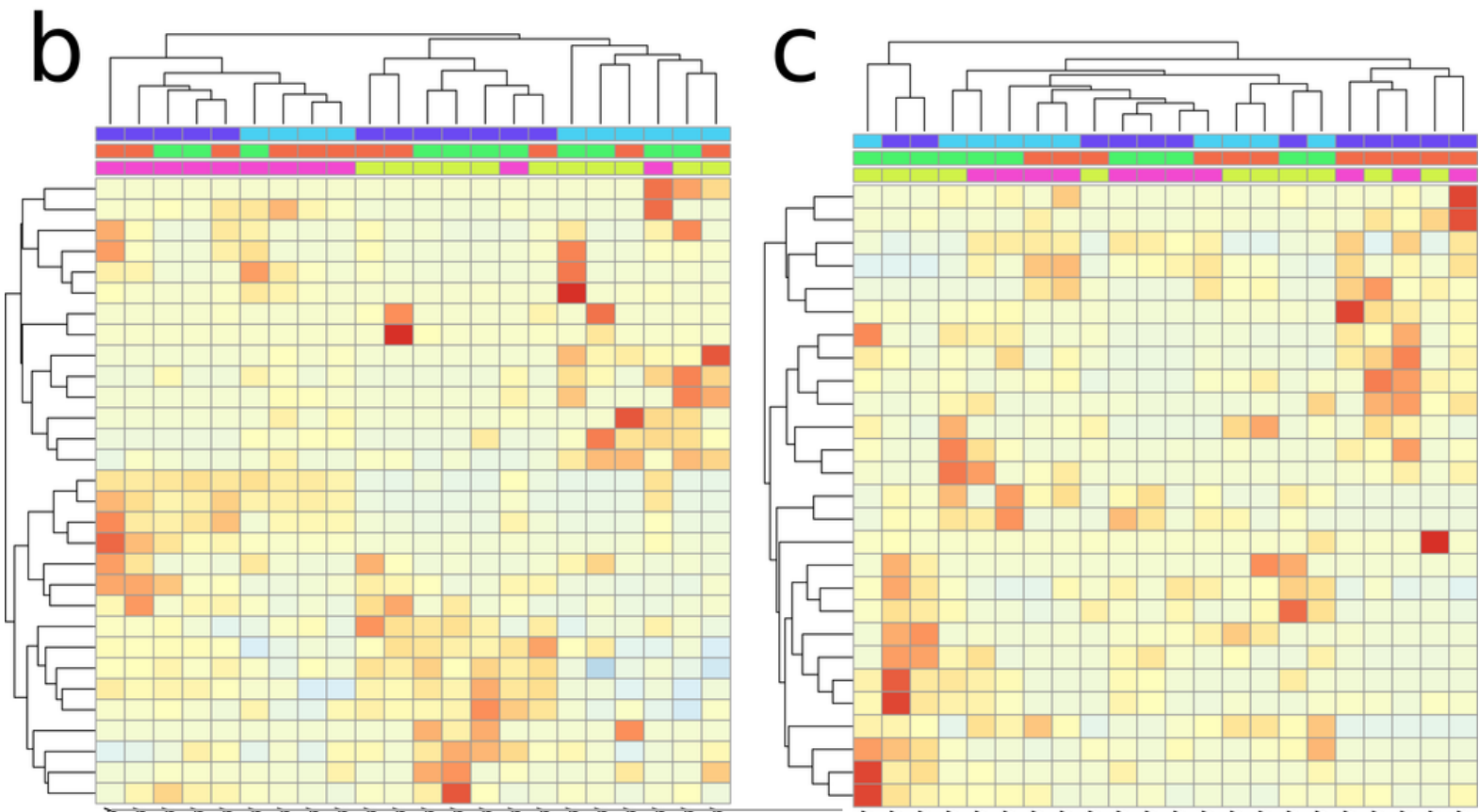

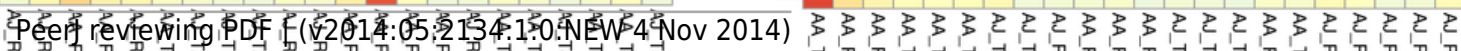

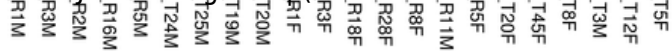




\section{Figure 3}

European and Asian samples are differentiated by their genotypic profiles

Based on mapping of sequencing reads to transcripts, 128,707 bi-allelic SNPs were called. Nematode samples were clustered into distinct clades transposing the genotype matrix to matrix of euclidean distances and analysing it with neighbour joining (A) and maximum parsimony (B) methods. Principal component analysis (PCA) was used to visualize the overall structure of the genotype data (C). The first principal component (PC1) explained $12 \%$ and the second component (PC1) $8 \%$ of the total variance in genotype differences according to the PCA eigenvalues (histogram inlay in C). PC1 clearly separates all Asian from all European nematode samples. 


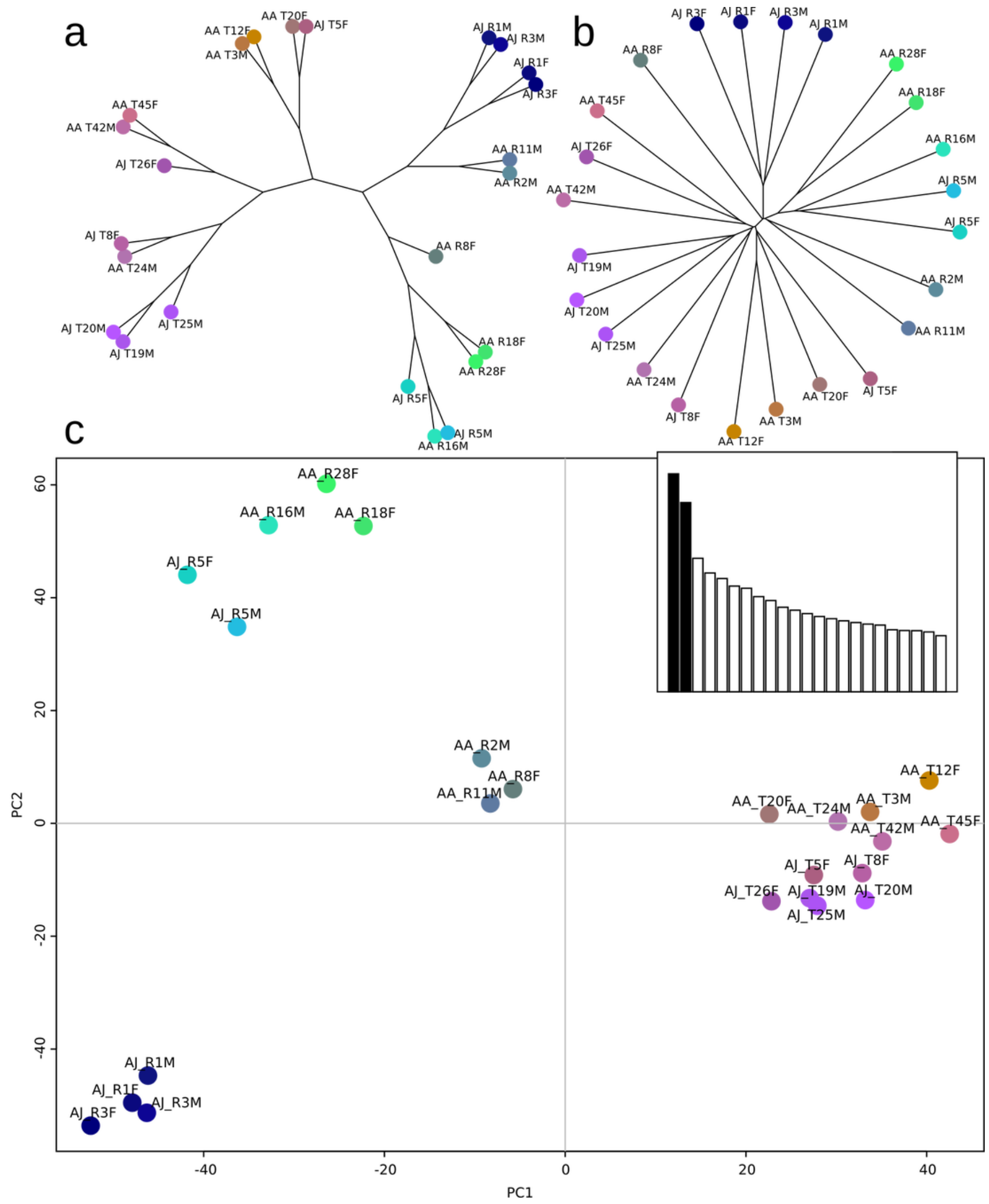




\section{Figure 4}

Gene ranking for contributions to genotypic differentiation

$\mathrm{K}$-means clustering of the principal components identified only two clusters in the genotyping data corresponding to Asian and European nematode samples respectively. Discriminant analysis of principal components then estimated a discriminant function maximising the between-group variance. Panel A displays the densities of individuals on the single retained discriminant function. This function was also used to rank loci according to their contribution to to genotypic discrimination. 589 loci with a contribution greater than $0.8 \times 10^{-5}$ are displayed in B and clustered based on Euclidean distances. 


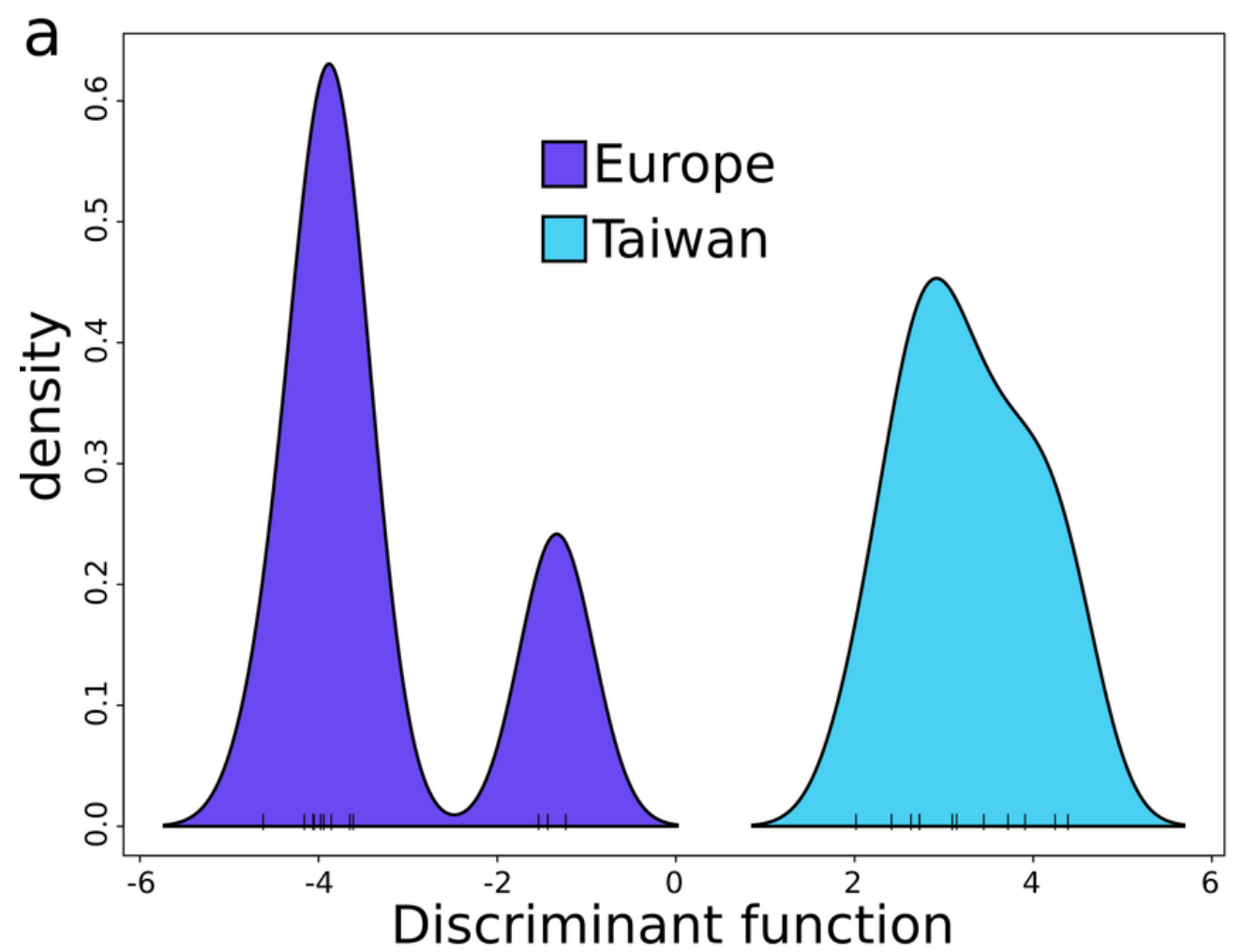

b

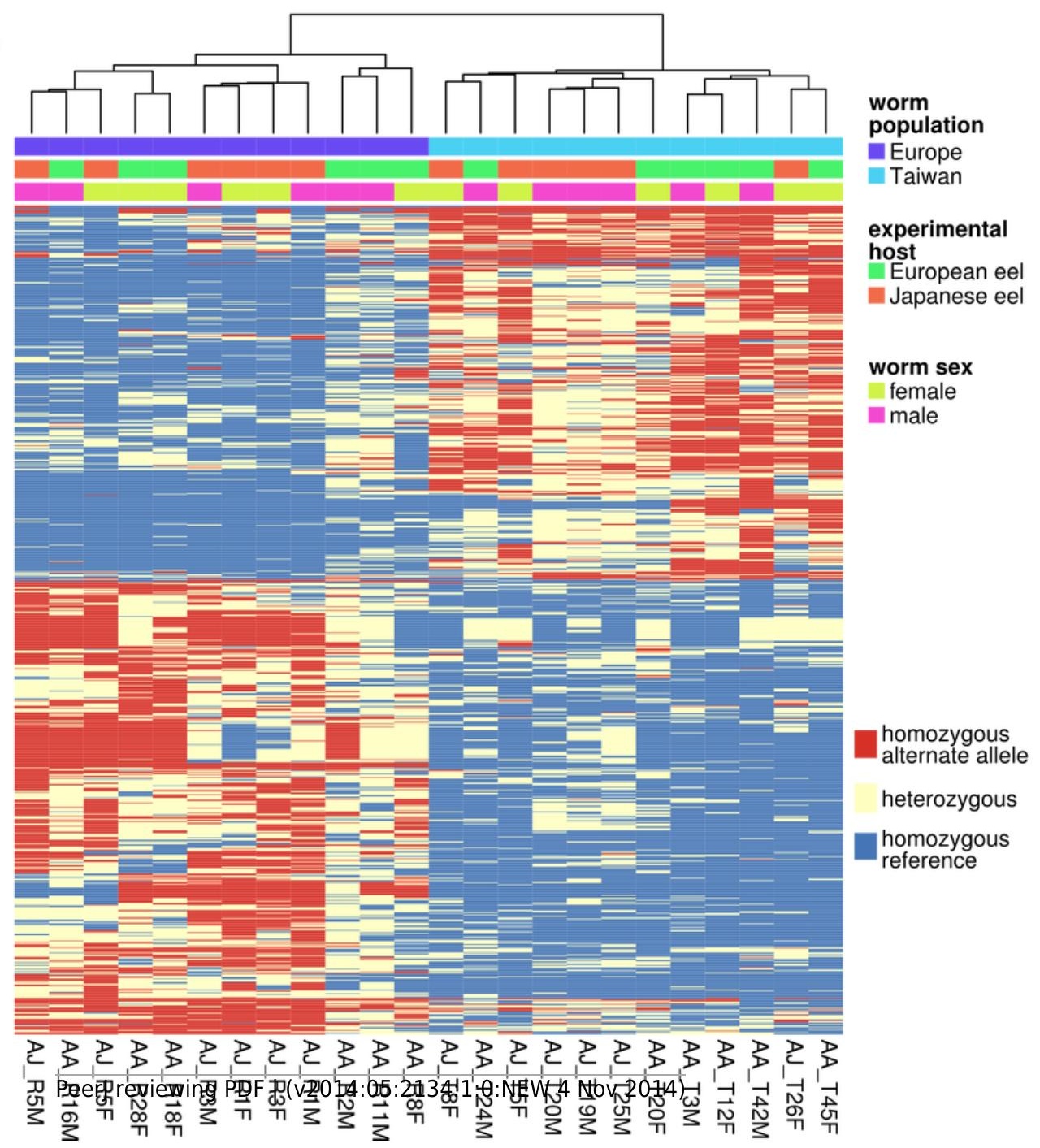

